\title{
Los Medios Sociales en la Promoción Turística de las Zonas Rurales
}

\section{Social Media in the Tourism Promotion of Rural Areas}

\section{María José Aguilar-Torres ${ }^{1}$, Eduardo Fabio Henriquez-Mendoza ${ }^{2}$, Franklin Gustavo Santín-Picoita ${ }^{3}$ y Hever} Sánchez-Martínez ${ }^{4}$ \section{RTED
- EDICIÓN: Pesiliencia
aradigmática}

Recibido: 15/agosto/202

Aceptado: 17/noviembre/2021

P Publicado: 29/noviembre/2021

Páginas: 191-199

\&aís

${ }^{1}$ Ecuador

${ }^{2}$ Ecuador

${ }^{3}$ Ecuador

${ }^{4}$ Ecuador

\section{IIIII Institución}

${ }^{1}$ Universidad Nacional de Loja ${ }^{2}$ Universidad Nacional de Loja ${ }^{3}$ Universidad Nacional de Loja ${ }^{4}$ Universidad Nacional de Loja

\section{Correo Eletrónico}

1maria.j.aguilar@unl.edu.ec ²eduardo.henriquez@unl.edu.ec ${ }^{3}$ franklin.santin@unl.edu.ec 4hever.sanchez@unl.edu.ec

\section{ORCID}

${ }^{1}$ https://orcid.org/0000-0002-8853-4570 ${ }^{2} \mathrm{https} / / /$ orcid.org/0000-0001-6102-9809 ${ }^{3} \mathrm{https} / / /$ orcid.org/0000-0002-3852-047X ${ }^{4} \mathrm{https}$ ://orcid.org/0000-0001-5189-5164

\section{Citar así: C A APA / IEEE}

Aguilar-Torres, M., Henriquez-Mendoza, E., Santín-Picoita, F. \& Sánchez-Martínez, H. (2021). Los Medios Sociales en la Promoción Turística de las Zonas Rurales Revista Tecnológica-Educativa Docentes 2.0, 1(1), 191-199. https://doi.org/10.37843/rted.v1i1.270

M. Aguilar-Torres, E. Henriquez-Mendoza, F. Santín-Picoita y H. Sánchez-Martínez, "Los Medios Sociales en la Promoción Turística de las Zonas Rurales ", RTED, vol. 1, n. $^{\circ} 1$, pp. 191-199, nov. 2021.

\section{Resumen}

El uso masivo de medios digitales ha creado nuevos canales para llegar a un número mayor de usuarios. Sin embargo, para tener resultados positivos es necesario implementar estrategias que logren un impacto significativo en sus audiencias y públicos objetivos. El objetivo de esta investigación fue analizar los medios digitales del Municipio de LojaEcuador con respecto a la promoción turística de sus zonas rurales. Metodológicamente se utilizó un enfoque mixto con una técnica del análisis de contenido en Facebook y Instagram, basada en publicaciones sobre promoción turísticas de las zonas rurales. Como criterio de selección para nuestra muestra se identificó publicaciones realizadas en los Fan Page y Perfil de las redes sociales Facebook e Instagram del Gobierno Autónomo Descentralizado Municipal de Loja, con un total entre 20 - 21 publicaciones sobre turismo de las zonas rurales respectivamente. Como resultado encontramos que hubo pocas publicaciones para promocionar los lugares turísticos de zonas rurales durante el 2019. En las discusiones y conclusión, se evidenció cómo el Departamento de Comunicación, promociona estas bondades de manera limitada en conjunto con la Jefatura de Turismo. Así como, las necesidades que hoy exige promocionar el turismo local, como el fortalecimiento en Comunicación en contenidos de interés para los usuarios.

Palabras clave: Redes sociales, turismo digital, turismo rural, parroquias rurales.

\section{Abstract}

The massive use of digital media has created new channels to reach a more significant number of users. However, to have positive results, it is necessary to implement strategies that substantially impact your target audiences and audiences. The objective of this research was to analyze the digital media of the Municipality of Loja-Ecuador concerning the tourism promotion of its rural areas. Methodologically was used a mixed approach with a content analysis technique on Facebook and Instagram, based on publications on tourism promotion in rural areas. As selection criteria for our sample, publications made on the Fan Page and Profile of the social networks Facebook and Instagram of the Autonomous Decentralized Municipal Government of Loja were identified, with a total between 20 - 21 publications on tourism in rural areas, respectively. As a result, we found few publications to promote tourist places in rural areas during 2019. In the discussions and conclusion, it was evidenced how the Department of Communication promotes these benefits in a limited way in conjunction with the Headquarters of Tourism and the needs that today require promoting local tourism, such as strengthening Communication in the content of interest to users.

Keywords: Social networks, digital tourism, rural tourism, rural parishes. 


\section{Introducción}

El uso masivo de medios digitales ha creado nuevos canales para llegar a un número mayor de usuarios. Sin embargo, para tener resultados positivos es necesario implementar estrategias que logren un impacto significativo en sus audiencias y públicos objetivos. Según Galarza "los medios digitales de comunicación permiten compartir: texto, audio, video y fotografías" (2015, p.7). El auge tecnológico impulsó mediante la comunicación, un turismo afianzado en los medios digitales, cuyo resultado es el posicionamiento global de los destinos turísticos y la interacción con millones de usuarios alrededor del mundo a través de contenido multimedia, reflexión compartida por Gutiérrez-Montoya, et al. (2018), quienes señalan que las Tecnologías de la información y la comunicación (TIC) amplían y posibilitan el desarrollo.

La comunicación turística se ha visto influenciada por los múltiples desarrollos tecnológicos. A su vez, los usuarios se han adaptado a nuevas formas de interacción hábitos y exigencias de consumo (Altamirano \& Túñez, 2014). Hoy, instituciones públicas emplean cada vez más redes sociales para promoción turística, pero desconocen su correcto manejo, limitando, su efectividad. En el turismo, las tecnologías son cada vez más utilizadas por empresas para promocionarse. Es necesario que las instituciones públicas tengan una visión más amplia del impacto que tienen esta información en redes sociales, así cómo saber usarlas para aumentar el flujo de turistas. Esto, delimitando e identificando información deseada por los usuarios que realmente aporte al conocimiento del destino, producto o servicio.

En este sentido, "la difusión de internet, las comunicaciones inalámbricas, los medios de comunicación digitales y una serie de herramientas de software social han provocado el desarrollo de redes horizontales de comunicación interactiva que conectan lo local y lo global en cualquier momento" (Castells, 2009, p. 101). Los medios digitales, se han desarrollado hasta crear una red de interacción y difusión de información de alcance mundial. Así estos medios digitales funcionan como redes de interacción social. Una oportunidad existente, gracias a las redes sociales y su constante crecimiento, son las plataformas de comercialización de productos, entro otros servicios como lo indica Sumosa "para tener éxito y crecimiento en las organizaciones, de acuerdo con la nueva realidad de la publicidad virtual, se necesita orientarse hacia los caminos donde regularmente los prospectos de clientes asisten y ahí comercializar los productos" (2019, p.07).

En la actualidad, los lugares que albergan mayores números de potenciales turistas (usuarios) son las plataformas digitales (Paniagua \& Assumpció, 2018, p. 226). Por ello, los medios digitales se han convertido en herramientas de promoción para las actividades turísticas que ofrecen alternativas diferentes en sitios o áreas rurales (Acerenza, 2006, p.41).

Loja cuenta con un sin número de atractivos, sean naturales o culturales, pero la escasa promoción se debe a que los sectores rurales están muy apartados (Fernández \& Miret, 2019). Sotomayor \& Cueva, (2020) señalan que "es una actividad que ha comenzado a tener relevancia". Sin embargo, en Ecuador el turismo rural es un fenómeno relativamente nuevo en nuestro país, mientras en otros países como Francia es una práctica de hace setenta años (Talón, \& Figueroa, 2009 , p.160). Esto se refleja limitadamente en la utilización de las redes sociales para promoción turística rural. Las estrategias digitales, no han sido aprovechadas al máximo o no se han aplicado adecuadamente sobre todo en instituciones públicas.

Por ende, la presente investigación tuvo como objeto de estudio las redes sociales del Gobierno Autónomo Descentralizado Municipal de Loja, Ecuador. Conociendo que los medios digitales son espacios web donde los usuarios pueden crear su propio contenido, entre ellos, se encuentran las redes sociales como Facebook e Instagram. Es así como, se tomó como objeto de estudio en la presente investigación a estas dos redes (FB y IG), para conocer el manejo, estrategias y plan social media utilizado por el departamento de 
comunicación.

\section{Metodología}

Para esta investigación, de corte cuantitativa-cualitativa, se utilizó el proceso deductivo, es decir, se inició con una revisión bibliográfica, que ayudó a razonar y explicar la realidad del objeto de estudio. Luego se partió de teorías generales hacia casos particulares. Algunos autores plantean ciertas incertidumbres de cara relación entre las partes cualitativa y cuantitativas involucradas en este tipo de investigación (Akerblad et al., 2020; Uprichard y Dawney, 2016). Pero, de acuerdo con Creswell \& Plano-Clark (2018), quienes justifican la pertinencia de esta mixtura, esta investigación se eligió integrar estos dos enfoques para dar respuesta integral a las variables principales planteadas.

Como criterio de selección para nuestra muestra se identificó publicaciones realizadas en los Fan Page y Perfil de las redes sociales Facebook e Instagram del Gobierno Autónomo Descentralizado Municipal de Loja, con un total entre 20 - 21 publicaciones sobre turismo de las zonas rurales respectivamente. Durante el desarrollo investigativo se realizó un análisis de los contenidos de los medios digitales del Municipio de Loja, Ecuador. Esto permitió la obtención de resultados mediante análisis estadístico, basándose en porcentajes.

El análisis de las publicaciones sobre promoción turísticas de las zonas rurales del año 2019 fue mediante el uso de la técnica del análisis de contenido, basados en el Facebook e Instagram en total fueron 21 fichas en Facebook y 20 fichas en Instagram. Se analizó los elementos de géneros usados, formatos audiovisuales utilizados $\mathrm{y}$ el número de parroquias promocionadas. Además, se realizaron entrevistas a los funcionarios del Gobierno Autónomo Descentralizado Municipal de Loja, llamados aquí como entrevistados, sobre el flujo informativo en las redes sociales del GAD Municipal en torno a la promoción turística de las parroquias rurales del cantón. También se entrevistaron a docentes y expertos que no pertenecían a la institución para obtener un mayor contraste.

\section{Resultados}

Al analizar los medios digitales del Gobierno Autónomo Descentralizado Municipal de Loja para su promoción turística de parroquias rurales se comprobó que la información se presenta de manera limitada, encontrando 21 publicaciones en Facebook y 20 en Instagram. Mediante fichas de observación se registró que el contenido de cada publicación era deficiente, escueto $\mathrm{y}$ limitado. En los hallazgos de formatos, textos, vínculos, hashtags $\mathrm{y}$ otras herramientas necesarias para la promoción, se evidenció una administración deficiente de respuestas oportunas a la hora de brindar información rápida a sus usuarios.

Los hallazgos también permitieron evidenciar que de las 13 parroquias rurales del cantón Loja, solo 6 de ellas tienen publicaciones en Facebook o Instagram a lo largo del 2019, denotando falta de promoción en redes sociales. El Departamento de Comunicación de la administración, promocionaba estas bondades en conjunto con la Jefatura de Turismo, desde estas instituciones, es donde nace la información para brindar promoción al turismo local.

Por todo lo anterior, hay que tener presente a la comunicación como nexo entre el destino y el turista. Porque su objetivo es "comunicar a los posibles turistas (consumidores) sobre una oferta turística. En el caso de la promoción de los destinos turísticos se identifican instrumentos de la promoción tradicional y no tradicional" (Castillo \& Castaño, 2015, p. 22). La comunicación en el ámbito del turismo colabora con los grupos de trabajo internos para "otorgar una calidad de servicios con sello propio que eleve los niveles de atención y se constituya como un factor de competencia a la hora de distinguir una empresa de otra o un destino de otro" (Palazzolo, 2016, p. 27).

En Instituciones públicas, como el Gobierno Autónomo Descentralizado Municipal de Loja, la difusión turística en redes sociales es coordinada por diferentes 
departamentos. Estas instituciones deben de tener presente que la promoción turística se habla de satisfacer las necesidades del consumidor desarrollando productos $\mathrm{y}$ servicios de acuerdo con la exigencia del turista (Castillo \& Castaño, 2015). Las empresas turísticas deben ofrecer más que la información de interés en sus sitios web y redes sociales; la oferta turística debe acompañarse con contenidos que complementen las necesidades de información del viajero. (Altamirano, Túñez \& Valarezo, 2016). En la revolución tecnológica, los medios sociales configuran un nuevo ecosistema informativo, caracterizado por la interactividad entre los prosumidores mediáticos y también los otros nuevos medios emergentes de la red (Suárez \& Cruz, 2016, p.81).

Por su parte Guallar et al. (2016) postulan que las redes sociales ofrecen múltiples servicios, desde la comunicación hasta el espacio adecuado para compartir noticias, informaciones de carácter público, incluso informaciones que son silenciadas en los medios tradicionales. Actualmente, las redes sociales como Facebook, Twitter, Instagram y YouTube son los aliados estratégicos de los medios de comunicación. Con estas plataformas los periódicos logran incidir en un mayor número de prosumidores. Luego de haber analizado las publicaciones referentes a turismo de las parroquias rurales del Gobierno Autónomo Descentralizado Municipal de Loja, Ecuador se detallan los resultados obtenidos.

\section{Análisis de las Fichas de Facebook}

En la Tabla 1, se muestra la cantidad de veces que una parroquia o zona rural ha tenido publicaciones en la página de Facebook del GADM de Loja. Los mayores porcentajes correspondieron a las parroquias de Vilcabamba con el 38\%, El Cisne con 29\% y Gualel con 24\%. Aunque San Pedro de Vilcabamba también tiene publicaciones su porcentaje es de $9,5 \%$ es muy bajo con respecto a las parroquias antes mencionadas. Sin embargo, se observa que de las 13 parroquias rurales del cantón Loja, solo 4 se han promocionado en cuanto turismo durante el año 2019.

\section{Tabla 1}

Número de Publicaciones por Parroquia

\begin{tabular}{lcc}
\hline PARROQUIA & FRECUENCIA & PORCENTAJE \\
\hline GUALEL & 5 & $23,8 \%$ \\
\hline EL CISNE & 6 & $28,6 \%$ \\
\hline VILCABAMBA & 8 & $38,1 \%$ \\
\hline SAN PEDRO & 2 & $9,5 \%$ \\
DE & & \\
VILCABAMBA & & $\mathbf{1 0 0 \%}$ \\
\hline TOTAL & $\mathbf{2 1}$ & \\
\hline
\end{tabular}

Nota. Facebook Gobierno Autónomo Descentralizado Municipal de Loja, elaboración propia (2021).

Los datos demuestran que las parroquias intentan generar turismo rural, porque dentro de sus redes sociales hubo actividad como: compartir texto, audio, video y fotografias (Galarza, 2015). Pero falta mayor iniciativa por parte de GADM de Loja. Solamente esa acción permitirá que la actividad comience a tener relevancia a nivel local e internacional. (Sotomayor \& Cueva, 2020).

En esta Tabla 2 se muestra la etiqueta y el tipo usado en cada publicación, se han determinado tres categorías: Corporativo, de Contenido y el No uso del mismo. El mayor porcentaje $47,61 \%$ presentado por el uso de hashtag Corporativo en relación con la GADM de Loja como Institución; seguido del $33,35 \%$ de publicaciones en las que no se utiliza la etiqueta y finalmente, solo el $19 \%$ usa hashtag del contenido de la publicación.

\section{Tabla 2}

Uso de Hashtag

\begin{tabular}{lll}
\hline HASHTAG & $\begin{array}{l}\text { FRECUENC } \\
\text { IA }\end{array}$ & $\begin{array}{l}\text { PORCENTA } \\
\text { JE }\end{array}$ \\
\hline DE & 4 & $19,04 \%$ \\
CONTENIDO & & \\
\hline $\begin{array}{l}\text { CORPORATI } \\
\text { VO }\end{array}$ & 10 & $47,61 \%$ \\
\hline NO & 7 & \\
\hline TOTAL & $\mathbf{2 1}$ & $33,35 \%$ \\
\hline
\end{tabular}

Nota. Facebook Gobierno Autónomo Descentralizado Municipal de Loja, elaboración propia (2021).

El uso de la etiqueta en redes sociales es una herramienta para que los usuarios puedan ubicar temas de su interés, así como para contactar a otros usuarios con intereses similares (Feixa et al. 2016). Así mismo, se 
utilizan para generar tendencia en determinados temas, generando posicionamiento de marca de la Institución si hablamos de hashtag corporativo. El posicionamiento y reconocimiento de destinos en el uso de contenido, si los utilizamos correctamente, para promoción turística podremos generar un mayor flujo de visitas en las cuenta y contenido de los medios sociales. Esto reflejará el aumento de turistas a determinados sitios con potencial turístico. Así lo ratifica Castelló-Martínez "la almohadilla (\#) permite clasificar y categorizar" (2013, p.1). Esta acción es beneficiosa, porque es más fácil llegar a viralizar el contenido turístico.

\section{Tabla 3}

\begin{tabular}{lcc} 
Uso de Vínculo & \\
\hline \multicolumn{2}{l}{ VÍNCULO } & FRECUENCIA PORCENTAJE \\
\hline PÁGINA WEB & 1 & $4,76 \%$ \\
\hline SITIOS & 0 & $0 \%$ \\
EXTERNOS & & $0 \%$ \\
\hline REDES & 0 & \\
SOCIALES & & \\
INSTITUCIONAL & & $23,81 \%$ \\
ES & & \\
\hline $\begin{array}{l}\text { REDES } \\
\text { SOCIALES }\end{array}$ & 5 & \\
EXTERNAS & & $71,43 \%$ \\
\hline NO & 15 & $\mathbf{1 0 0} \%$ \\
\hline TOTAL & $\mathbf{2 1}$ & \\
\hline
\end{tabular}

Nota. Facebook Gobierno Autónomo Descentralizado Municipal de Loja, elaboración propia (2021).

En la Tabla 3 la etiqueta vínculo fue usado en 6 publicaciones de promoción turística de las parroquias o zonas rurales de Loja, de ellos, cinco que son el $23,81 \%$ ha sido en referencia de redes sociales externas a la Institución. La mayor parte de ellas enlazan a redes sociales personales del alcalde del cantón, denominadas como Jorge Bailón. Tan solo una direccionaba a la página web del GADM de Loja. Sin embargo, el 71,43\% de las publicaciones no registraron el uso del hipervínculo.

Los hipervínculos se utilizan para aquellos usuarios que necesitan más información de la que se expone en la publicación. Además, puedan encontrarla en otras páginas web, lo adecuado es redirigirlo a la página web institucional donde puedan encontrar más información, no a páginas de figuras públicas que poco aporte darán a las mismas. El poco uso de esta herramienta muestra poco interés por ampliar la información que se difunde a la ciudadanía, usar vínculos puede dar a los turistas datos relevantes para conocer los lugares turísticos en la zona rural del cantón. Es necesario considerar, sobre todo en esta nueva era digital que el "los usuarios de hashtags emiten más valoraciones" (Castello-Martínez, A., 2013).

\section{Análisis de las Fichas de Instagram}

\section{Tabla 4}

Número de Publicaciones por Parroquia PARROQUI FRECUENCIA PORCENTAJE A

\begin{tabular}{lll}
\hline MALACATOS & 3 & $15 \%$ \\
\hline GUALEL & 8 & $40 \%$ \\
\hline EL CISNE & 2 & $10 \%$ \\
\hline VILCABAMBA & 4 & $20 \%$ \\
\hline SAN PEDRO & 2 & $10 \%$ \\
DE & & \\
VILCABAMBA & & $5 \%$ \\
\hline SAN LUCAS & 1 & $\mathbf{1 0 0 \%}$ \\
\hline \multicolumn{1}{c}{ TOTAL } & $\mathbf{2 0}$ & \\
\hline
\end{tabular}

Nota. Facebook Gobierno Autónomo Descentralizado Municipal de Loja, elaboración propia (2021).

La Tabla 4 representa la cantidad de veces que una parroquia rural tuvo publicaciones en la página de Instagram del GADM de Loja. Los mayores porcentajes correspondiente a las parroquias de Gualel, Vilcabamba y Malacatos respectivamente. A diferencia de Facebook, observó que otras parroquias tuvieron menor contenido a lo largo del 2019. Sin embargo, estas parroquias como Gualel, Vilcabamba y Malacatos mantuvieron porcentajes bajos para tener una promoción turística que arrojara resultados positivos.

La Promoción turística en redes sociales requiere de publicaciones constantes para mantener a los usuarios interesados en las actividades a realizar en los destinos turísticos que queremos posicionar. "Por su propia naturaleza, están interrelacionados con un conjunto de empresas y disciplinas, dedicadas todas a servir a una clientela formada por turistas, para establecer un conjunto de 
servicios turísticos en un entorno natural, social, cultural, económico, tecnológico y político" (Ascanio, 2017, p.24). El bajo número de publicaciones anuales sobre el turismo en las parroquias rurales por parte del Municipio de Loja, no son suficientes para cumplir con el objetivo de dar a conocer los lugares turísticos de Loja.

\section{Tabla 5}

\begin{tabular}{lcc}
$\begin{array}{l}\text { Uso de Hashtag } \\
\text { HASHTAG }\end{array}$ & FRECUENCIA & PORCENTAJE \\
\hline $\begin{array}{l}\text { DE } \\
\text { CONTENIDO }\end{array}$ & 4 & $20 \%$ \\
\hline CORPORATIVO & 14 & $70 \%$ \\
\hline NO & 2 & $10 \%$ \\
\hline \multicolumn{1}{c}{ TOTAL } & $\mathbf{2 0}$ & $\mathbf{1 0 0 \%}$ \\
\hline
\end{tabular}

Nota. Facebook Gobierno Autónomo Descentralizado Municipal de Loja, elaboración propia (2021).

La Tabla 5 presenta la etiqueta del tipo de contenido que se ha usado en cada publicación determinado en tres categorías: Corporativo, de Contenido y el No uso del mismo. El 70\% de las publicaciones la etiqueta es corporativo fue utilizado con el objetivo de potenciar los eventos del GADM de Loja. Sin embargo, el uso de redes sociales como Instagram genera flujo de usuarios hacia el contenido de interés, es más fácil que los turistas encuentren información si esta tiene las referencias que otorgan este tipo de herramientas, en el caso de las publicaciones analizadas solo el $20 \%$ utiliza hashtag de contenido o de relevancia en cuanto al turismo de cada parroquia.

\section{Tabla 6}

\section{Uso de Vínculo}

\begin{tabular}{lcc}
\hline VÍNCULO & FRECUENCIA & PORCENTAJE \\
\hline PÁGINA WEB & 2 & $10 \%$ \\
\hline SITIOS EXTERNOS & 1 & $5 \%$ \\
\hline $\begin{array}{l}\text { REDES SOCIALES } \\
\text { INSTITUCIONALES }\end{array}$ & 1 & $5 \%$ \\
$\begin{array}{l}\text { REDES SOCIALES } \\
\text { EXTERNAS }\end{array}$ & 1 & $5 \%$ \\
\hline NO & 15 & $75 \%$ \\
\hline \multicolumn{1}{c}{ TOTAL } & $\mathbf{2 0}$ & $\mathbf{1 0 0} \%$
\end{tabular}

Nota. Facebook Gobierno Autónomo Descentralizado Municipal de Loja, elaboración propia (2021).

La Tabla 6 presenta la etiqueta del uso de enlaces o hipervínculos como poco frecuente en publicaciones de Instagram, como se puede observar en la figura el $75 \%$ no tiene enlaces. Sin embargo, cuando se trata de promoción turística en redes sociales el turista, sobre todo el turista 2.0, busca información relevante que no encuentra en redes sociales, información complementaria que ayude a responder a las dudas antes de viajar.

Por otro lado, el $10 \%$ de los vínculos utilizados en las publicaciones sobre promoción turística de las parroquias rurales dirigen al inicio de la página web del GADM de Loja, donde el turista no puede encontrar información relevante. Lo mismo sucede con los vínculos a redes sociales externas, ya que solo llevan al perfil del alcalde de Loja, conocido como Jorge Bailón. "Un sector con una enorme y variada oferta y con una demanda potencial... precisa de la existencia de grandes sistemas de intermediación, que antes eran de carácter físico pero que, con la llegada de Internet, han sufrido una transformación radical" (Fundación Orange, 2016, p.10). Con la llegada de las redes sociales, el turismo sufrió una transformación, la aparición del turista 2.0 exige de los servicios experiencias de mayor nivel.

Eso se logra únicamente con el uso de las herramientas que generan las redes sociales, e incluso de las necesidades que los turistas demandan ver en los medios digitales.

\section{Discusión}

Al analizar los medios digitales del Gobierno Autónomo Descentralizado Municipal de Loja para la promoción turística de parroquias o zonas rurales se comprobó que su promoción turística de parroquias o zonas rurales es limitad. Porque, Se encontraron 21 publicaciones en Facebook y 20 en Instagram. Las fichas de observación permitieron analizar el contenido de cada publicación, demostrando las debilidades en el formato, texto, vínculos, hashtags y otras herramientas necesarias para su promoción. Los hallazgos también permitieron evidenciar que de las 13 parroquias rurales del cantón Loja, solo 6 de ellas tuvieron publicaciones en Facebook o Instagram a lo largo del 2019, 
denotando la falta de promoción redes sociales.

Las entrevistas realizadas a expertos evidenciaron varias falencias como la limitada información de las instituciones estudiadas que no permiten una adecuada promoción turística, como la falta de planificación en cuanto al contenido publicado en las diferentes redes sociales. Así como, la falta de contenido para las parroquias que tienen atractivos turísticos pero que por desconocimiento no tienen un flujo importante de turistas. Al determinar las herramientas que se usan en los medios digitales del Gobierno Autónomo Descentralizado Municipal de Loja se determina que las redes sociales ofrecen materiales básicos de medición de información y contenido demandado para mejorar la interactividad.

Al conocer la audiencia y la interactividad que provocan los productos de comunicación en los medios digitales del Gobierno Autónomo Descentralizado Municipal de Loja, se comprobó que los encuestados detallaban información como la frecuencia con la que observaban publicaciones referentes a la promoción turística de parroquias o zonas rurales de Loja, interacción en las publicaciones, interés hacia el mismo. De ello se observó, poca satisfacción de los encuestados frente a las publicaciones del GADM de Loja, donde el $54 \%$ de los encuestados no tuvo interés por interactuar en estas publicaciones.

En el Municipio de Loja, para medir el impacto de sus publicaciones utilizan herramientas básicas como las estadísticas de Facebook y cuadro de mando de Excel como publicaciones más y menos demandadas, horas pico, contenido básico para mejorar la interactividad. La Institución no cuenta con estadísticas turísticas de redes sociales debido a que generan contenido de 17 direcciones, la mayor parte de ello, institucional. De acuerdo con la entrevistada es más "valioso para el usuario y nosotros publicamos en lo que más piden".

Uno de los mayores problemas es no contar con una red social enfocada en la creación de contenido turístico. De acuerdo con la entrevistada Docente del Departamento de Ciencias de la Comunicación de la Universidad Técnica Particular de Loja, se reafirmó la idea de que las redes sociales en el ámbito turístico son necesarias para lograr una promoción turística, ya que tienen una incidencia en las nuevas tecnologías porque el nuevo turista busca información. En el caso de Facebook e Instagram, tienen un posicionamiento importante debido al número de usuarios que existen en nuestra ciudad, además que son plataformas visuales en fotografía y video son necesarias para que los turistas interactúen con el contenido publicado.

De acuerdo con la entrevistada, las parroquias rurales tienen un potencial que debe ser expuesto no por parroquia sino por destino turístico, con información que realmente sea necesaria para el turista con el objetivo para motivar la su visita, es decir, primero se debe analizar el comportamiento de nuestros turistas para, con base en ello, crear el contenido para redes sociales. En función de la información recabada a través de los intereses del turista, se debe publicar la información, para ello la planificación semanal es importante para lograr un flujo alto de turistas, al menos con cuatro días de anticipación.

De acuerdo con nuestra profesional entrevistad, el Municipio de Loja debería crear una cuenta en redes sociales únicamente para la promoción turística de Loja, para evitar que la información institucional se mezcle con la de promoción turística. Con una estrategia de contenidos para potencializar el destino turístico a través de contenidos de valor.

\section{Conclusiones}

De acuerdo con análisis del contenido de las redes sociales Facebook e Instagram del Municipio de Loja se observó que existen pocas publicaciones para promocionar los lugares turísticos de las parroquias rurales durante el 2019. Considerando que la institución ha realizado 21 y 20 publicaciones respectivamente en Facebook e Instagram y de ellas, solo se promocionó a seis de las trece 
parroquias rurales que tiene el cantón. Por ello, es necesario crear un plan de contenido para redes sociales, con ello aumentará el número de publicaciones relacionadas a la promoción turística de las parroquias rurales de Loja, afianzado en un cronopost para abarcar el mayor número de atractivos turísticos rurales.

Las publicaciones realizadas para la promoción turística de las parroquias rurales no utilizan los elementos necesarios para informar a los turistas 2.0, ni permiten acceder a la información complementaria sobre los destinos turísticos; como lo harían con el uso adecuado de hashtag e hipervínculos y recursos multimedia. Solo el $1 \%$ de las publicaciones realizadas por la entidad utilizan herramientas para aumentar el flujo de interactividad e interés de los usuarios y potenciales turistas.

El contenido publicado en redes sociales para la promoción turística de las parroquias rurales del GADM de Loja, es de carácter informativo lo que quiere decir que les da mayor relevancia a eventos realizados por la Institución más que a los atractivos turísticos de las parroquias rurales. Debido a que la Institución tiene cuentas en las redes sociales Facebook e Instagram para todos sus Departamentos, la información turística es escasa y se no adquiere relevancia entre las informaciones que genera la entidad.

Pese a la cantidad de equipo humano y técnico con el que cuenta el departamento de comunicación, así como el alcance de las redes sociales del GADM de Loja, no existe personal dedicado a la promoción de los destinos turísticos de las parroquias rurales de Loja.

No existe un plan estratégico para la publicación del contenido referente a la promoción turística de las parroquias rurales, las publicaciones realizadas durante el 2019 no tienen una periodicidad establecida, ni cuentan con un formato determinado que las distinga de otras publicaciones institucionales. De ahí que, es necesaria la coordinación con la Jefatura de Turismo para realizar un mapa de los atractivos turísticos de las parroquias rurales para crear contenido que sea atractivo para los usuarios de redes sociales, con productos comunicacionales como videos, fotorreportajes e historias con datos específicos para uso del turista.

\section{Referencias}

Akerblad, L. Seppänen-Järvelä, R. y Haapakoski, K. (2021). Integrative Strategies in Mixed Methods Research. Journal of Mixed Methods Research, 1-19. https://doi.org/10.1177/1558689820957125

Acerenza, M. (2006). Conceptualización, Origen y Evolución del Turismo.

Altamirano, V. \& Túñez, M. (2016). Promoción y difusión turística de los países de Iberoamérica a través de los medios sociales. International Journal of Information Systems and Tourism (IJIST), 1(1), 7690.

http://www.uajournals.com/ojs/index.php/ijist/articl e/view/122

Ascanio, A. (2017). Teoría del Turismo. Trillas.

Castells, M. (2013). Comunicación y Poder. https://n9.cl/w9ik

Castello-Martínez, A. (2013). El uso de Twitter como canal de información y conversación por parte de los medios convencionales. Introducción a la Investigación de Medios Publicitarios. https://rua.ua.es/dspace/bitstream/10045/26898/1/A raceli_Castello_Martinez_1.pdf

Castillo, M. \& Castaño, V. (2015). La promoción turística a través de técnicas tradicionales y nuevas. Red de Revistas Científicas de América Latina, el Caribe, España y Portugal, volumen, 24. 737-757. https://www.redalyc.org/pdf/1807/180739769017.pd f

Castelló-Martínez, A. (2013). El uso de hashtags en Twitter por parte de los programas de televisión españoles. In I Congreso Internacional de Comunicación y Sociedad Digital. UNIR-Universidad Internacional de La Rioja.

Creswell, J.W. \& Plano, V.L. (2018). Designing and conducting mixed methods research. Sage.

Fundación Orange. (2016). La Transformación digital en el sector turístico. Evoca http:/www.fundacionorange.es/wpcontent/uploads/ 2016/05/eE_La transformacion_digital_del_sector turistico.pd $\bar{f}$

Fernández, A. M., y Miret, F. C. (2019). La gastronomía como recurso de la identidad ancestral: el caso de la Parroquia Chuquiribamba, Loja, Ecuador. Revista Amazónica Ciencia y Tecnología, 8(2), 126-135.

Feixa, C., Fernández-Planells, A., \& Figueras-Maz, M. (2016). Generación Hashtag. Los movimientos juveniles en la era de la web social. Revista Latinoamericana de Ciencias Sociales, Niñez y Juventud, 14(1), 107-120. 
Galarza, V. (2015). Origen y evolución de los medios digitales en el norte del Ecuador. Pontificia Universidad Católica del Ecuador. https://www.researchgate.net/publication/30785574 9_Origen_y_evolucion_de_los_medios_digitales_en _el_norte_del_Ecuador

Gutiérrez-Montoya, G. A., Sánchez-Jiménez, M. Á., \& Galiano-Coronil, A. (2018). Redes sociales como medio de promoción turística en los países iberoamericanos. RETOS. Revista de Ciencias de la Administración y Economía, 8(15), 135-150.

Guallar, J., Suau, J., Ruiz, C., Sáez, A., \& Masip, P. (2016). Redistribución de noticias y debate público en las redes sociales. El profesional de la información, 25(3), 358-366.

Paniagua, F. J., \& Huertas, A. (2018). El contenido en los medios sociales de los destinos turísticos y la búsqueda de información de los usuarios. Cuadernos De Turismo, https://doi.org/10.6018/turismo.41.327131

Palazzolo, S. (2016). La Comunicación en el Turismo. Ugerman Editor.

Sumosa, R. (2019). Turismo Comunitario, Turismo Digital, Turismo Informacional, Turismo Inteligente. Revista Turydes: Turismo y Desarrollo, 26. https://www.eumed.net/rev/turydes/26/turismotipos.html

Sotomayor, A. O., \& Cueva, P. A. O. (2020). Ambiente, sociedad y turismo comunitario: La etnia Saraguro en Loja-Ecuador. Revista de ciencias sociales, 26(2), 180-191.

Suárez, C., \& Cruz, J. (2016). Los dilemas deontológicos del uso de las redes sociales como fuentes de información. Análisis de la opinión de los periodistas de tres países. Revista Latina de Comunicación Social, 71, 66-84.

Talón, P. \& Figueroa, C. (2009). Nuevas tendencias y retos en el sector turismo: un enfoque multidisciplinar. Delta.

Uprichard, E. \& Dawney, L. (2016). Data Diffraction: Challenging Data Integration in Mixed Methods Research. Journal of Mixed Methods Research, 13(1), 19-32.

https://doi.org/10.1177/1558689816674650 